\section{Polarizing influence of TCRs}

The polarization of helper $\mathrm{T}$ cells is thought to be shaped mainly by the cytokine milieu. In Immunity, Germain and colleagues use intravital imaging to understand the influence of $T$ cell antigen receptor (TCR) signaling strength and interactions with antigen-presenting dendritic cells (DCs) on the polarization of helper $T$ cells. Adjuvants known to drive $T$ helper type $1\left(T_{H} 1\right)$ responses elicit higher expression of the costimulatory molecule CD80 on DCs and longer DC-T cell interaction times. However, strong peptide stimulation via the TCR also selectively drives $T_{H} 1$ polarization and, furthermore, this trumps the influence of the adjuvant. Thus, strong TCR signaling results in $T_{H} 1$ cells even with an otherwise ' $\mathrm{T}_{\mathrm{H}}$ 2-biasing' adjuvant. TCR signaling of strong intensity upregulates expression of the $\beta 2$ chain of the receptor for interleukin 12 (IL-12), which makes T cells receptive to IL-12 and $T_{H} 1$ biasing. The integration of TCR signals and cytokine signals therefore proceeds in a hierarchical manner.

Immunity 41, 63-74 (2014)

\section{Preexisting microclusters}

TCR microclusters are defined structures that contain a variety of signaling molecules and are involved in the activation of T cells. In the Journal of Immunology, Varma and colleagues find that TCR microclusters preexist even in truly naive cells with transgenic TCR expression that have never encountered agonist ligand. These preexisting microclusters contain a constant number of TCRs and key T cell signaling adaptors (such as Grb2 and Lat) and, furthermore, exclude the receptor phosphatase CD45. Thus, the microclusters have many of the structural and compositional characteristics required for the effective activation of $\mathrm{T}$ cells. It remains unclear how these ligand-indpendent microclusters are initially formed, but their preexistence may help facilitate the rapid signaling observed in T cells after they encounter high-affinity agonist peptide.

J. Immunol. 193, 56-67 (2014)

\section{Mechanisms of population dynamics}

T cell populations require mechanisms to create a dynamic antigen-response range independently of the initial population size. In eLife, Tkach et al. show that crosstalk between signaling via the TCR and the receptor for IL-2 (IL-2R) and feedback over the time of antigen exposure enable $T$ cell populations of various sizes to respond to a large range of stimuli. TCR signaling inhibits phosphorylation of the transcription factor STAT5 in a manner dependent on the antigen dose (but independent of IL-2R), while phosphorylated STAT5 inhibits IL-2 production but upregulates IL-2Ra expression. In this regulatory-loop setting, inhibition of IL-2 signaling by the TCR is critical for 'scaling' of IL-2 production dependent on the antigen dose but independent of population size. This study suggests that the accumulation of IL-2 is 'scaled' as a function of antigen load and represents a means of reporting that load independently of the number of T cells in the starting monoclonal or polyclonal population. eLife (9 April 2014) doi:10.7554/eLife.01944

Written by Laurie A. Dempsey, Zoltan Fehervari \& Ioana Visan

\section{IL-23 in negative selection}

After expression of the $\alpha \beta T C R$, induction of the transcription factor ROR $\gamma$ t promotes the survival of early $\mathrm{CD} 4^{+} \mathrm{CD} 8^{+}$double-positive (DP) thymocytes, but its downregulation in late DP cells is required for maturation to the $\mathrm{CD} 4^{+}$or $\mathrm{CD}^{+}$single-positive stage. In Nature Communications, Mountz and colleagues show that late-stage DP thymocytes are susceptible to IL-23-induced upregulation of ROR $\gamma \mathrm{t}$ expression and subsequent apoptosis. IL-23 induced by infection with Aspergillus fumigatus enhances the negative selection of thymocytes but has no effect on positive selection. IL-23 induces expression of the IL-23 receptor on late DP thymocytes but not on early DP thymocytes and acts through the receptor to upregulate ROR $\gamma \mathrm{t}$ expression and trigger apoptosis. Stimulation via the TCR is required for IL-23-induced apoptosis, which suggests that IL-23 signaling is secondary to an antigendependent signal in self-reactive thymocytes. These results correlate with the observation that the thymus becomes much smaller during the infection of mice with A. fumigatus.

Nat. Commun. (8 July 2014) doi:10.1038/ncomms5259

\section{Triggering $\mathrm{mTORC} 1$ in T cells}

T cells proliferate in response to strong agonist-induced stimulation of the TCR and costimulatory signaling via CD28. This process is known to depend on the cellular metabolic regulator kinase mTORC1. In Science Signaling, Kane and colleagues show that the activation of $\mathrm{mTORC} 1$ in $\mathrm{CD} 4^{+} \mathrm{T}$ cells in response to TCR signaling involves the adaptor Carma 1 and the paracaspase Malt1 but, unexpectedly, not the associated adaptor Bcl-10 or their downstream effector kinase IKK. Loss of Carma1 or Malt1 or inhibition of the catalytic activity of Malt1 in T cells results in their inability to activate mTORC1-dependent signaling pathways in response to stimulation via the TCR plus CD28 but not by other activation pathways. The activation of $\mathrm{mTORC} 1$ is impaired after inhibition of the kinase PKC, which activates the Carma1Malt1 complex. Inhibition or loss of Malt1 or Carma 1 also blunts $T$ cell proliferation and the metabolic switch from oxidative phosphorylation to glycolysis in response to TCR stimulation. Thus, a PKC-Carma1-Malt1-dependent pathway is required for the activation of mTORC1 in response to signaling via the TCR. LAD Sci. Signal. (10 June 2014) doi:10.1126/scisignal.2005169

\section{Novel innate $\mathrm{PLZF}^{+} \alpha \beta$ T cells}

The transcription factor PLZF is required for the development of several innate-like populations of thymus-derived T cells. In the Journal of Immunology, two studies by Berg and colleagues identify and characterize a previously unknown $\mathrm{PLZF}^{+}$population of TCR $\alpha \beta^{+} \mathrm{T}$ cells that arise in mice deficient in the TEC kinase Itk, which regulates TCR signaling. This T cell population, which differs from invariant natural killer T cells, MAIT cells and $\gamma \delta$ T cells, has a diverse TCR $\alpha$ repertoire and does not require either $\beta_{2}$-microglobulin or major histocompatibility complex class II molecules for thymic development. The thymic development of these cells does require interactions between the SLAM family of adhesion factors and the adaptor SAP. However, CD4 ${ }^{+} \mathrm{PLZF}^{+} I t k^{-/-}$thymocytes and their $\mathrm{CD}^{+}$counterparts seem to have different requirements for development. These cells 'preferentially' home to the spleen and gut, where their numbers are influenced by the microbiome. Thus, Itk activity seems to regulate the formation of a distinct subset of innate T cells. LAD J. Immunol. 193, 673-687 \& 688-699 (2014) 